\title{
THE POISSON TENDENCY IN TRAFFIC DISTRIBUTION ${ }^{1}$
}

\author{
By Leo Breiman \\ University of California, Los Angeles
}

1. Introduction. This note is concerned with models for one-way flow of traffic on the infinite line. A simple and frequently used model is: at $t=0$, points on the line (automobiles) are selected in accord with a Poisson process. Then velocities are assigned to these points, independently for each point and independent of the position of the point, drawn from the same parent distribution. The points then maintain their assigned velocities at all times. If a fixed point on the line is selected, then times between successive arrivals of autos at this point are, according to the above model, independent random variables with a negative exponential distribution. This result is hardly in agreement with experiment. Frank Haight, of the U.C.L.A. Traffic Engineering Department, suggested to the writer, a model in which the velocity assignment mechanism is retained, but introducing more general initial distributions. The purpose of this note is to show that in a strong sense the Poisson distribution is the only initial distribution leading to a "stable" traffic flow. Our main result is that for a wide class of initial distributions, the passage of time brings a convergence to the Poisson distribution.

This result was announced at the Congress of the International Statistical Institute, September 1961, [1]. At that time Alan Miller of Birmingham University, and George Weiss of the University of Maryland informed me that they had each independently obtained similar results [2], [3]. After some discussion we established that their proofs held only for initial distributions such that the gaps between points were independent and identically distributed, and that they had both used analytic methods of proof, which differed considerably from our approach. Since the present proof is short and relatively uncomplicated, it may have some merit.

It is a pleasure to acknowledge the pleasant and illuminating conversations on this subject with Frank Haight.

2. The Model. At $t=0$, let there be a set of starting points $X_{1}, X_{2}, \cdots$ on the negative real axis which are obtained as observations of a stochastic point process. Concerning this process we assume

(a) A spatial density $\sigma$ exists with probability one, i.e.,

$$
\lim _{x \rightarrow \infty}\left\{\text { no. of } X_{k} \text { in }[0,-x]\right\} / x=\sigma,
$$

with probability one.

(b) There is no "clumping up" of autos, i.e., for any finite interval $I$, the ex-

Received February 21, 1962; revised September 25, 1962.

1 This research was supported in part by the Information Systems Branch of the Office of Naval Research under Contract Nonr-222(53). 
pected number of $X_{k}$ in $I$ can be bounded above by $M$, where $M$ depends only on the length of $I$.

Associated with $X_{k}$ is $V_{k}$, its velocity. The $V_{k}$ are assumed independent of each other and of the $X_{1}, X_{2}, \cdots$ random variables, with common distribution $G(v)=P\left(V_{k}<v\right), G(v)=0, v \leqq 0$. Further,

(c) $G(v)=\int_{0}^{v} g(u) d u$, where $g(u)$ is almost everywhere continuous (w.r. to Lebesque measure) and bounded on every finite interval.

Denote by $X_{k}(t)$ the position of the $k$ th auto after time $t, X_{k}(t)=$ $X_{k}+t V_{k}$, and let $N_{t}(I)$ be the number of $X_{k}(t)$ in the interval $I$, where $|I|<\infty,(|I|=$ length of $I)$. Then we prove.

Theorem. Under (a), (b), (c) above, for fixed $I, j$,

$$
\lim _{t \rightarrow \infty} P\left(N_{t}(I)=j\right)=\left(\lambda^{j} / j !\right) e^{-\gamma}, \quad \text { where } \lambda=\sigma|I| .
$$

3. Prelude to the Proof. A Poisson convergence theorem is necessary which differs only slightly from the classical: consider for every $n$ an infinite sequence of trials $Z_{1}^{(n)}, Z_{2}^{(n)}, \ldots$ which are independent for fixed $n$ and result in either success or failure, with $P\left(Z_{k}^{(n)}=S\right)=P_{k}^{(n)}, P\left(Z_{k}^{(n)}=F\right)=1-P_{k}^{(n)}$. Let $N_{n}$ be the total number of successes in the $n$th sequence.

Theorem. If

$$
\begin{aligned}
& \sum_{k=1}^{\infty} P_{k}^{(n)} \rightarrow \lambda \text { as } n \rightarrow \infty \\
& \sup _{k} P_{k}^{(n)} \rightarrow 0 \text { as } n \rightarrow \infty
\end{aligned}
$$

then for fixed $j$,

$$
\lim _{\imath} P\left(N_{n}=j\right)=\left(\lambda^{j} / j !\right) e^{-\lambda} .
$$

The proof is immediate (see, for example, [4] pp. 263-264).

4. The Proof. We will compute $P\left(N_{t}(I)=j \mid X_{1}, X_{2}, \cdots\right)$ by noticing that for fixed $X_{1}, X_{2}, \cdots$, the $X_{k}(t)$ are independent. We will count a success if $X_{k}(t) \varepsilon I$, otherwise a failure. Applying the result of the above paragraph, if

$$
\begin{aligned}
& \lim _{t \rightarrow \infty} \sum_{k} P\left(X_{k}(t) \varepsilon I \mid X_{1}, X_{2}, \cdots\right)=\lambda \\
& \lim _{t \rightarrow \infty} \sup _{k} P\left(X_{k}(t) \varepsilon I \mid X_{1}, X_{2}, \cdots\right)=0
\end{aligned}
$$

then

$$
\lim _{t \rightarrow \infty} P\left(N_{t}(I)=j \mid X_{1}, X_{2}, \cdots\right)=\left(\lambda^{j} / j !\right) e^{-\lambda} .
$$

Because, even though $t$ is a continuous parameter the above theorem will yield the desired limit law for every sequence $t_{n} \rightarrow \infty$. Suppose (i) and (ii) above hold true for all values of $X_{1}, X_{2}, \cdots$ with the possible exception of a set of probability zero, where $\lambda$ is a constant not depending on $X_{1}, X_{2}, \cdots$. Then the resulting limit law is true for all values of $X_{1}, X_{2}, \cdots$ again with the possible exception of a set of probability zero. Now, we may take the expectation of both 
sides of the limit law and using the boundedness of the left-hand side (since it is a probability) apply the Lebesque bounded convergence theorem to prove the desired result. For any interval $J=\left[v_{1}, v_{2}\right)$, define $G[J]$ as $G\left(v_{2}\right)-G\left(v_{1}\right)$. With this

$$
P\left(X_{k}+t V_{k} \varepsilon I \mid X_{1}, \cdots\right)=P\left(V_{k} \varepsilon\left[\left(I-X_{k}\right) / t\right] \mid X_{1} \cdots\right)=G\left[\left(I-X_{k}\right) / t\right],
$$

and

$$
\cdot \sup _{k} G\left[\left(I-X_{k}\right) / t\right] \leqq \sup _{u} G(I / t+u)
$$$$
-\infty<u<+\infty \text {. }
$$

If the expression on the right does not go to zero as $t \rightarrow \infty$, there exists a sequence of intervals $J_{1}, J_{2}, \cdots$ such that $\left|J_{n}\right| \rightarrow 0$, but $G\left(J_{n}\right) \rightarrow 0$. Since any continuous distribution function is uniformly continuous on the whole line, this is impossible.

As for (i) suppose, initially, that there is a $v_{0}$ such that $g(v)=0, v>v_{0}$. Let $M_{t}(y)=\left\{\# X_{k}\right.$ in $\left.(0,-t y), y \geqq 0\right\}$, and note that

$S_{t}=\sum_{k} P\left(X_{k}(t)_{\varepsilon} I \mid X_{1}, \cdots\right)=\sum_{k} G\left[\left(I-X_{k}\right) / t\right]=\int_{0}^{\infty} G(I / t+y) d M_{t}(y)$.

Place $m_{t}(y)=M_{t}(y) / t$, getting $S_{t}=\int_{0}^{\infty} t G(I / t+y) d m_{t}(y)$. The idea is now simple; if $g(v)$ is continuous, $g(v)=0, v>v_{0}$, then $G(I / t+y) /(|I| / t)$ converges uniformly to $g(y)$ and is zero for $y>v_{0}+\epsilon, t$ sufficiently large. On the other hand, $m_{t}(y) \rightarrow \sigma y$, with probability one (and the same exceptional set for all $y$ ). Therefore,

$$
\lim _{t} S_{t}=|I| \sigma \int g(y) d y=|I| \sigma .
$$

If $g(v)$ is not continuous, then by (c), for $\epsilon>0$, there exists a $v_{1}>v_{0}$ and continuous functions $g^{-}(v), g^{+}(v)$ such that they vanish for $v>v_{1}, g^{-}(v) \leqq g(v)$ $\leqq g^{+}(v)$ and

$$
\int_{0}^{v_{1}}\left(g^{+}(u)-g^{-}(u)\right) d u \leqq \epsilon .
$$

Placing $G^{-}(v)=\int_{0}^{v} g^{-}(u) d u, G^{+}(v)=\int_{0}^{v} g^{+}(u) d u$, we have

$$
G^{-}(I / t+y) \leqq G(I / t+y) \leqq G^{+}(I / t+y)
$$

therefore,

$$
\int t G^{-}(I / t+y) d m_{t} \leqq S_{t} \leqq \int t G^{+}(I / t+y) d m_{t} .
$$

By the former discussion, the left and right hand sides converge to $\sigma|I|$ $\cdot \int g^{-} d y, \sigma|I| \int g^{+} d y$ respectively, and since these are bounded below and above by $\sigma|I|(1 \pm \epsilon)$, the result follows. 
Finally, there remains the problem of disposing of the cutoff point $v_{0}$. For an arbitrary $G(v)$ satisfying $c)$, and any $\epsilon>0$, take $v_{0}$ such that $1-G\left(v_{0}\right) \leqq \epsilon$. Define another assignment of probabilities $V_{k}^{\prime}$ by

$V_{k}^{\prime}=V_{k}$ if $V_{k} \leqq v_{0}$,

$V_{k}^{\prime}=Y_{k}$ if $V_{k}>v_{0}$ where $Y_{k}$ is selected independently from the uniform distribution on $\left[0, v_{0}\right]$,

and let $N_{t}^{\prime}(I)$ be the number of autos in $I$ after time $t$ under the velocities $V_{k}^{\prime}$. Note that the event $N_{t} \neq N_{t}^{\prime}$ occurs only if, for at least one $k$, either one or both of $A_{k}, A_{k}^{\prime}$ occur, where

$$
\begin{aligned}
& A_{k}: X_{k}(t) \varepsilon I, X_{k}^{\prime}(t) \varepsilon^{\prime} I \\
& A_{k}^{\prime}: X_{k}(t) \varepsilon^{\prime} I, X_{k}^{\prime}(t) \varepsilon I .
\end{aligned}
$$

Hence

$$
\begin{gathered}
P\left(N_{t} \neq N_{t}^{\prime}\right) \leqq \sum_{k} P\left(A_{k} \cup A_{k}^{\prime}\right) \leqq \sum_{k} P\left(A_{k}\right)+\sum_{k} P\left(A_{k}^{\prime}\right) \\
P\left(A_{k}\right) \leqq P\left(X_{k}(t) \varepsilon I, V_{k}>v_{0}\right)=\int_{v_{0}}^{\infty} P\left(X_{k}+v t \varepsilon I\right) d G(v) \\
\sum_{k} P\left(A_{k}\right) \leqq \sum_{k} \int_{v_{0}}^{\infty} P\left(X_{k} \varepsilon I-v t\right) d G(v)=\int_{v_{0}}^{\infty}\left[\sum P\left(X_{k} \varepsilon I-v t\right)\right] d G(v) .
\end{gathered}
$$

The expression $\sum_{k} P\left(X_{k} \varepsilon I-v t\right)$ is the expected number of $X_{k}$ in the interval $I-v t$, and by (b) is bounded by $M$, not depending on $v t$. Thus $\sum_{k} P\left(A_{k}\right)$ $\leqq M \epsilon$. For the second sum $P\left(A_{k}^{\prime}\right) \leqq P\left(X_{k}^{\prime}(t) \varepsilon I, V_{k}>v_{0}\right)=P\left(X_{k}+Y_{k} t \varepsilon I\right)$ $\cdot P\left(V_{k}>v_{0}\right)$. This yields

$$
\sum_{k} P\left(A_{k}^{\prime}\right) \leqq \varepsilon \sum_{k} 1 / v_{0} \int_{0}^{v_{0}} P\left(X_{k} \varepsilon I-v^{\prime} t\right) d v^{\prime}
$$

and using again $\sum_{k} P\left(X_{k} \varepsilon I-v^{\prime} t\right) \leqq M$, gives $\sum_{k} P\left(A_{k}^{\prime}\right) \leqq M \epsilon$. Putting things together, $P\left(N_{t} \neq N_{t}^{\prime}\right) \leqq 2 M \epsilon$ from which follows

$$
\left|P\left(N_{t}=j\right)-P\left(N_{t}^{\prime}=j\right)\right| \leqq 2 M \epsilon .
$$

Using our former result, $\lim _{t} P\left(N_{t}^{\prime}=j\right)=\lambda^{j} / j ! e^{-\lambda}, \lambda=\sigma|I|$, and since $\epsilon$ is arbitrary, the limit relation also holds for $P\left(N_{t}=j\right)$.

The writer is indebted to the referee for a correct proof of the result $P\left(N_{t} \neq N_{t}^{\prime}\right) \leqq 2 M \epsilon$, and other helpful comments.

\section{REFERENCES}

[1] Breiman, L. (1961). On some probability distributions occurring in traffic flow. To be published in Proc. 30th Congress, Internat. Statist. Inst., Paris.

[2] MrLuer, A. On the theoretical justification of the random queues model for road traffic flow. Submitted to Proc. Cambridge Philos. Soc.

[3] Herman, R. and Weiss, G. (1962). Statistical properties of low density traffic. Quart. Appl. Math. 20 121-130.

[4] Feluen, W. (1957). Probability Theory and its Applications. (2nd ed.). Wiley, New York. 